\title{
Kinetics of Hydrochloric Acid Leaching of Copper from its Ore
}

\author{
Yousef Mubarak \\ Department of Chemical Engineering, American University of Beirut, Lebanon, ym26@aub.edu.lb
}

\begin{abstract}
The effects of some experimental parameters on the copper leaching process were investigated and presented in a previous article, while in the current article kinetic models to represent the effects of leaching temperature, acid concentration, and ore particle size on the leaching rate will be examined and analyzed. The obtained results showed that none of the surface chemical reaction or the pore diffusion on its own can control the whole copper dissolution process. The mixed kinetics model which includes two different leaching processes including the surface chemical reaction and diffusion through a porous product layer was found to be suitable for describing and fitting the experimental data up to $65 \%$ copper dissolution $(X)$ at a high stirring speed of 1000 $\mathrm{rpm}$. The apparent activation energies for pore diffusion and surface reaction were estimated to be 12.26 and 9.61 $\mathrm{kcal} / \mathrm{mol}$, respectively. Values of surface reaction rate constant in the range from $6.35 \times 10^{-4}$ to $5.49 \times 10^{-3} \mathrm{~cm} / \mathrm{sec}$ and pore diffusion coefficient in the range from $3.58 \times 10^{-8}$ to $2.36 \times 10^{-7} \mathrm{~cm}^{2} / \mathrm{sec}$ have been obtained.
\end{abstract}

Key words: Leaching, Copper Ore, Shrinking Core, Surface Reaction, Pore Diffusion, Mixed Kinetics

\section{INTRODUCTION}

One of the most widely used metals in various industries is copper which is found in nature as oxide and sulfide ores [1, 2]. Due to the global increase in demand, low-grade oxides as well as high-grade ores are considered as main sources of copper. To eliminate some of the negative environmental and economical impacts of pyrometallurgical methods such as high initial investment costs, high energy requirements, and the generation of environmentally hazardous SO gases, and the incentive to development metal extraction processes with reduced environmental impact, researchers have focused on the development of hydrometallurgical processes that may be alternatives to conventional pyrometallurgical methods [3]. On the other hand, pyrometallurgical methods are not suitable for the processing of low-grade ores and this can be considered as another reason to recover copper from lowgrade ores by hydrometallurgical methods [4]

In previous studies $[5,6]$, the effects of leaching temperature, acid concentration, particle size, stirring speed, and solid/liquid ratio on the leaching process of copper ore using hydrochloric acid as the main lixiviant were studied and presented. The present work quantitatively investigates the kinetic mechanism of the leaching process of copper in hydrochloric acid solution, hence a theoretical background for the available heterogeneous reaction models will be presented here for heterogeneous reactions between fluid A and solid B, which may be represented by $A_{(\text {fluid })}+v_{B} B_{(\text {solid })} \rightarrow$ Products, where $v_{B}$ is the stoichiometric factor (number of moles of metal reacted per mole of lixiviant).

A single model can't incorporate all the features of liquid/solid reactions. In fact, different classes of models have been postulated and used to describe these systems.

\subsection{Shrinking Core Model}

The shrinking core model (SCM), is one of the earliest models used and is well described in standard textbooks on chemical reaction engineering $[7,8]$. The model is mainly applicable to nonporous or relatively less porous solids and assume that the reaction occurs at a sharp interface that separates the reacted outer shell (product or "ash" layer) and the unreacted inner core of the solid. This model was first developed in 1955 by Yagi and Kunii [9], Figure 1, who presented five steps occurring in succession during a reaction:

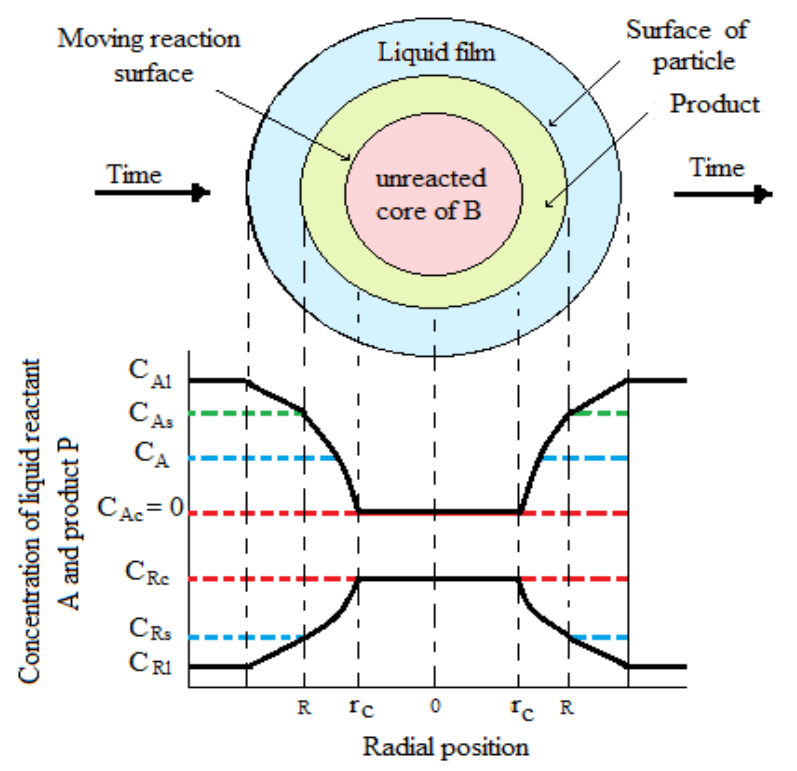

Figure 1: Representation of concentrations of reactants and products for the reaction $A_{l}+v_{B} B_{s} \rightarrow P$ for a particle of unchanging size 
Step 1. Diffusion of liquid reactant A through the liquid film surrounding the particle to the surface of the solid.

Step 2. Penetration and diffusion of A through the blanket of products to the surface of the unreacted core.

Step 3. The reaction of liquid A with the solid at this surface.

Step 4. Diffusion of liquid products through the products layer back to the exterior surface of the solid.

Step 5. Diffusion of liquid products through the liquid film back into the main body of fluid.

For single-step, irreversible reactions, the main steps involved are diffusion through the fluid film, diffusion through the product layer, and reaction at the interface [10]. For the shrinking core model, thus, there are three resistances which may control the overall rate of reaction, as discussed below:

\subsubsection{Diffusion through Liquid Film Controls}

Whenever the resistance of the liquid film controls, the concentration profile for reactant A will be as shown in Figure 2. From this figure, it is seen that no reactant is present at the surface of the particle; hence the concentration driving force is constant during the reaction of the particle.

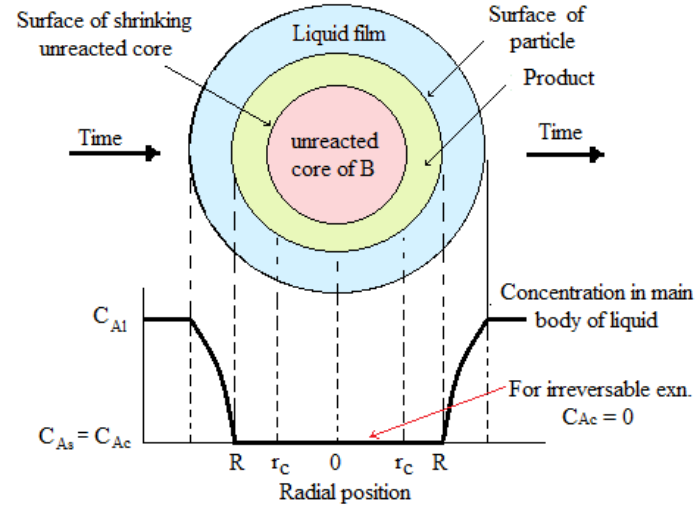

Figure 2: Representation of a reacting particle when diffusion through the liquid film is the controlling resistance

So, if the agitation in the reactor is not sufficient there will be significant boundary layer control the rate of the reaction according to the following equations [10]:

$\frac{t}{\tau}=X, \quad$ where $\tau=\frac{\rho_{B} R_{0}}{3 v_{B} k_{L} C_{A_{L}}}$

where $\tau$ is the time for complete reaction of a particle under the controlling step, $k_{L}$ is the mass transfer coefficient, $R_{o}$ is the initial particle radius, $\rho_{B}$ is the molar density of solid reactant, and $C_{A_{L}}$ is the concentration of species $\mathrm{A}$ at the liquid.

\subsubsection{Diffusion through Product Layer Controls}

Figure 3 illustrates the situation in which the resistance to diffusion through the product layer controls the rate of reaction, and the integrated form of this model is [7]:

$\frac{t}{\tau}=1-3(1-X)^{\frac{2}{3}}+2(1-X)$, where $\tau=\frac{\rho_{B} R_{0}{ }^{2}}{6 v_{B} D_{\mathrm{e}} C_{A_{L}}}$ where $D_{e}$ is the effective diffusion coefficient in a porous structure. By substituting $\tau$ into the equation:

$\frac{6 v_{B} D_{\mathrm{e}} C_{A_{L}} t}{\rho_{B} R_{0}^{2}}=1-3(1-X)^{\frac{2}{3}}+2-2 X$

\subsubsection{Chemical Reaction Controls}

Illustration of concentration gradients within a particle when a chemical reaction control is presented in Figure 4. Since the progress of the reaction is unaffected by the presence of any products layer, the quantity of material reacting increases as the available surface of unreacted core increases. The final form of the equation describing this model is [7]:

$\frac{t}{\tau}=1-(1-X)^{\frac{1}{3}}, \quad$ where $\tau=\frac{\rho_{B} R_{0}}{v_{B} K_{S} C_{A_{L}}}$

where $K_{S}$ is the surface reaction rate constant. By substituting $\tau$ in Eq. (4):

$\frac{v_{B} K_{S} C_{A_{L}} t}{\rho_{B} R_{0}}=1-(1-X)^{1 / 3}$

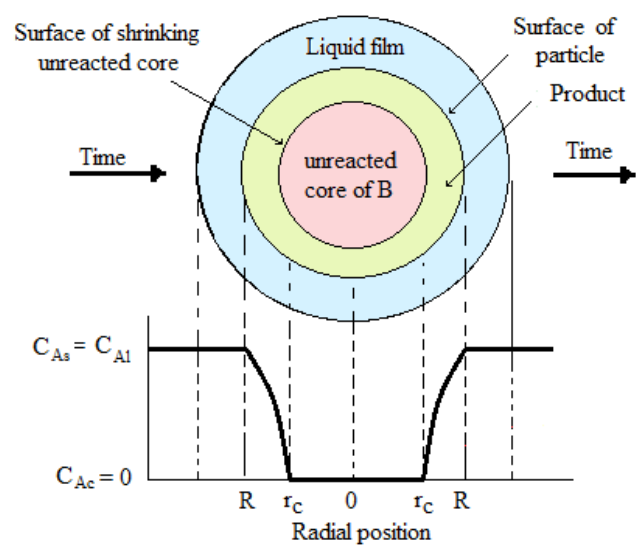

Figure 3: Representation of a reacting particle when diffusion through the product layer is the controlling resistance

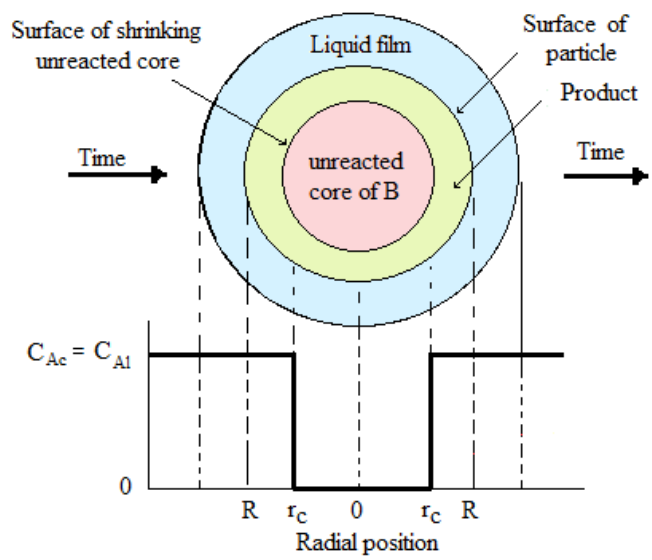

Figure 4: Representation of a reacting particle when a chemical reaction is a controlling resistance

\subsection{Uniform Conversion Model}

When the solid is porous, the liquid can penetrate the solid and the reaction may now be assumed to take place all over 
the volume of the particle, Figure 5, rather than at a sharp interface [11].

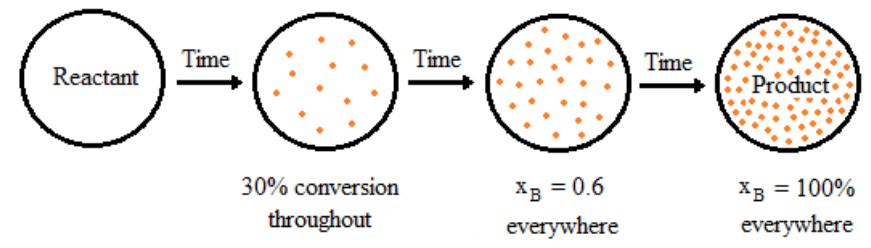

Figure 5: Representation of a reacting particle when the uniform conversion model is controlling

In general, the rate of reaction at the interior points would be lower than that at the surface due to the concentration gradient. In the special situation when no diffusion resistance exists, the reaction occurs uniformly all through the particle, leading to the so-called homogenous model [10]. This homogenous model has not been applied to solid-liquid reactions except for some special cases [11].

\subsection{Grainy Particle Model}

In the basic grainy particle model (GMP), also referred to as the particle-pellet model, it is assumed that the reaction of moderately porous solid particles is represented by the reaction of particles consisting of several grains of solid, the grains are spherical and are of the same size. It is further assumed that each grain reacts according to SCM with either product layer, diffusion, or chemical reaction control and that the size of the grains does not change with reaction; thus, there is no change in particle voidage. A diffusion resistance for the liquid species exists in the interparticle spaces within the particle [10], as seen in Figure 6.

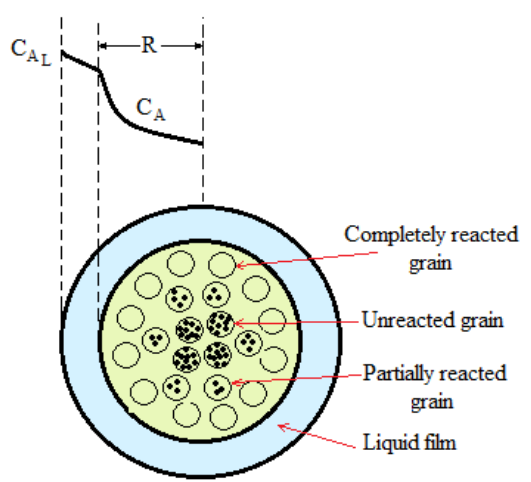

Figure 6: Schematic representation of the grainy particle model.

The GMP is a two-parameter model whose characteristic times are:

$\tau_{\text {grain }}=$ time for complete conversion of a grain surrounded by a uniform concentration of reactant $\mathrm{A}$.

$\tau_{\text {diffusion }}=$ time for complete conversion of a particle by diffusion if $\tau_{\text {grain }}$ is equal to zero. Thus, $\tau_{\text {diffusion }}$ is the complete conversion time of the particle if diffusion of liquid reactant between the grains is the rate-controlling step.
At the extreme, where $\tau_{\text {diffusion }}<<\tau_{\text {grain }}$ thus negligible interparticle diffusion resistance, all the grains react away at the same time and in the same way. Here the uniform conversion model or SCM with either surface reaction or product layer controlling steps may be observed. At the opposite extreme where $\tau_{\text {diffusion }}>>\tau_{\text {grain }}$, the observed rate is similar to that of the shrinking core model with product layer diffusion control [11].

\subsection{Crackling Core Model}

Park and Levenspiel [12] proposed the so-called crackling core model, Figure 7, to account for the S-shaped behavior of $X$ versus $t$ plots observed in some systems. In this model, the reaction is assumed to occur in two steps

$$
\begin{gathered}
B_{(\text {nonporous })} \rightarrow B_{(\text {porous })} \\
B_{(\text {porous })} \rightarrow \text { Product }
\end{gathered}
$$

The model envisages two stages, each of which is characterized by a certain characteristic time, and the model, therefore, involves two parameters:

$\tau_{\text {grain }}=$ time required for complete conversion of a grain.

$\tau_{\text {core }}=$ time required for the original nonporous particle to become completely grainy porous solid.

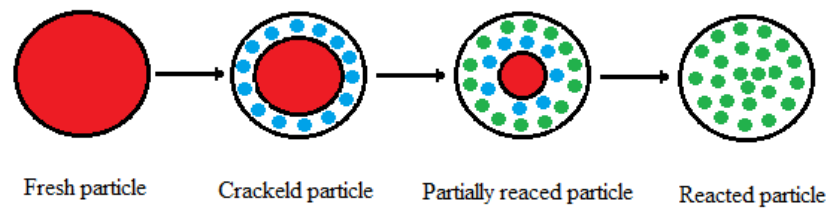

Figure 7: Basic stages in a crackling core model

In the limiting case of fast crackling ( $\tau_{\text {grain }}>>\tau_{\text {core }}$ ), the controlling processes shift over to individual grains and one observes a model characterized by the reaction of individual grains. The individual grains follow the shrinking cored model with either reaction or product layer diffusion controlling, the conversion-time relationship becomes independent of the particle dimensions. In the other extreme of slow crackling, ( $\tau_{\text {grain }}<<\tau_{\text {core }}$ ), the processes within the grain proceed much faster, and for the pellet as a whole, one observes shrinking core behavior with reaction control $[10$, 11].

The basic feature of this model is that it predicts the S-shaped curve observed experimentally in some systems. Also, if $\tau_{\text {grain }}$ $\rightarrow \infty$, the crackling proceeds faster, rendering the particle porous and taking it to some intermediate level of conversion within a not extremely large time. Subsequent conversion to $100 \%$, however, is extremely difficult to be observed because of large $\tau_{\text {grain }}[11]$.

\subsection{Hsu and Murr Model}

A simple kinetic model was developed by Hsu and Murr [13], based on a special form of the shrinking core model and assuming control by chemical reaction. The integrated form of the model is: 
$1-(1-X)^{\frac{1}{3}}=\frac{1}{v_{B} K R_{0}} * \operatorname{Ln}\left(v_{B} k K C_{A} t+1\right)$

where $C_{A}$ is the lixiviant concentration, $k$ is the rate constant parameter, $K=\frac{k}{D}$ is the surface reaction rate constant, and $D$ is the diffusion coefficient in the product layer.

\subsection{Mixed Kinetics Model}

The mixed kinetics model proposed by Wadsworth [14], involves steady-state diffusion of the reactant through the reacted portion of the ore fragment followed by a chemical reaction within the reaction zone. The leaching reaction occurs at the site of included minerals that are present in veinlets or as discrete disseminated particles. Reactants and products of the leaching must, therefore, be transported in solution-filled channels within the ore fragment. The model also assumes that the circulation of the leach solution around the particle is sufficient to maintain liquid reactant concentration so that bulk solution transport is not a ratecontrolling step. Thus, the kinetics are then limited only by processes occurring within the ore fragments.

Figure 8 represents an idealized ore particle showing the reaction zone of thickness $\delta_{w}$. The final form of the equation is [15]:

$$
\begin{aligned}
& 1-\frac{2}{3} X-(1-X)^{\frac{2}{3}}+\beta\left[1-(1-X)^{\frac{1}{3}}\right]=\gamma C_{A} t \\
& \text { where } \beta=\frac{2 D}{R_{0} K_{S}} \text {, and } \gamma=\frac{2 D}{v_{B} R_{0}^{2}}
\end{aligned}
$$

A statistical method can be used for computing the parameters $\beta$ and $\gamma$. Using the measured values of $\beta$ and $\gamma$ and using their defining equations values of $D$ and $K_{S}$ can be determined.

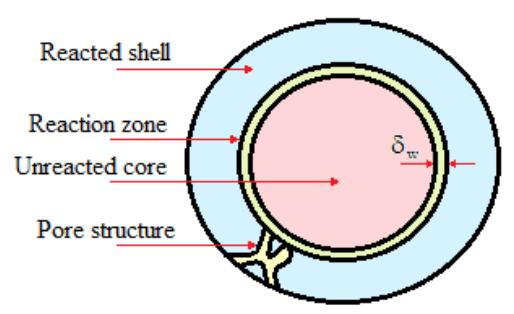

Figure 8: Representation of mixed kinetics model

\subsection{Previous Studies}

Bai et al. [16] studied the kinetics of copper leaching from low-grade cuprite ores using a sulfuric acid solution. Using 0.125-0.074 mm ore particles, about $92.5 \%$ of copper was achieved at a reaction temperature of $353 \mathrm{~K}$ for $180 \mathrm{~min}$ reaction time with $150 \mathrm{~g} / \mathrm{dm}^{3}$ sulfuric acid and a solid/liquid ratio of 1:15. The authors claimed that the shrinking core model complies with the obtained leaching kinetic experimental data. Using a $13 \%$ sulfuric acid concentration, stirring speed of $600 \mathrm{rpm}$, liquid/solid ratio of $10 \mathrm{~mL} / \mathrm{g}$, and $50{ }^{\circ} \mathrm{C}$ as reaction temperature, Azizi et al. [17] managed to dissolve about $91 \%$ copper content after 80 min leaching time. The dissolution kinetics was examined according to heterogeneous models and found that the dissolution of copper in sulfuric acid solution is controlled by the diffusion through the product layer considering the shrinking core model to be appropriate enough to describe the leaching process.

The leaching kinetics of malachite in perchloric acid solutions was investigated by Tanaydin and Demirkiran [18]. The mixed kinetics control model was found to be the most appropriate model that describes the rate of leaching reaction. In another study, Demirkiran et al. [19] found that copper leaching from a malachite ore in acetic acid solutions fits the mixed kinetic control model accounting for diffusion through the product layer at temperatures between $20^{\circ} \mathrm{C}$ and $45{ }^{\circ} \mathrm{C}$ and a surface chemical reaction at temperatures between 45 ${ }^{\circ} \mathrm{C}$ and $60{ }^{\circ} \mathrm{C}$. Using sulfuric acid under controlled conditions, the leaching of dolomitic-copper ore was studied by Ntengwe [20]. Copper recovery was found to be 72 to 93 $\%$ for $75 \mu \mathrm{m}$ particle size and 65 to $84 \%$ for the $212 \mu \mathrm{m}$ particle size. The reaction of the ore with $\mathrm{H}_{2} \mathrm{SO}_{4}$ is reported to be second order with respect to chalcopyrite and first order with respect to dolomite and was consistent with the shrinking core model.

The leaching kinetics of a complex copper ore using ammonia was examined by Ghosh et al. [21]. About $83 \% \mathrm{Cu}$ could be selectively extracted under the following conditions: $125-212$ $\mu \mathrm{m}$ particle size, $120{ }^{\circ} \mathrm{C}, 1.29 \mathrm{~mol} / \mathrm{L} \mathrm{NH}_{3}$, and $2.5 \mathrm{hr}$ as leaching time. The leaching process was found to be surface reaction controlling. Reilly and Scott [22] proposed an electrochemical surface reaction model with a cathodic reduction of oxygen on the solid surface as the ratedetermining step for the leaching kinetics of chalcopyrite in $\mathrm{NH}_{3}$ medium.

Although many experimental studies have been performed to gain a better understanding of the leaching process of copper ores and its operation, there is still a lack of information concerned with the aim of kinetics modeling. An accurate understanding of leaching kinetics helps to interpret the complex behavior of the leaching process. The main objective of this study is to examine copper dissolution kinetics based on heterogeneous reaction models, and the best-fitted equation to the experimental data will be determined.

\section{MATERIALS AND METHODS}

\subsection{Equipment and Sampling}

A one-liter nominal capacity Pyrex glass rounded bottom flask with three necks carrying a thermometer, a stirrer, and a sampling device was used to run the leaching experiments [6]. The Teflon stirrer used consists of a $25 \mathrm{~cm}$ long shaft of $1 \mathrm{~cm}$ diameter with two $3 \times 0.5 \times 1 \mathrm{~cm}$ blades at its end. The stirrer is driven by a variable speed electric motor and can give a maximum stirring speed of $1000 \mathrm{rpm}$. The reactor temperature was maintained at the desired temperature within $\pm 0.5^{\circ} \mathrm{C}$ using a stirred water bath. 
The sampling device is calibrated to deliver $5 \mathrm{~mL}$ solution and has a $42 \mu \mathrm{m}$ fritted glass end for filtering the reaction solution. To allow obtaining five samples during the first two minutes of the reaction, the sampling device is connected to a vacuum pump that decreases the time required for drawing off and delivering liquid samples. By manipulated a 2-way and 3way valves to appropriate positions, drawing off or delivery of the sample can be achieved easily. The details of both, the experimental setup and the sampling device, are presented in detail in reference 6 .

\subsection{Materials}

The copper ore used in the current study has been supplied by the Natural Resources Authority-Jordan from Wadi Araba. The ore is whitish beige, medium-hard, medium crystalline, and fractured dolomite with copper disseminations. After crushing, grounding, and sieving, three sizes were used to conduct the leaching experiments. The average particle sizes used are 462,1100 , and $1850 \mu \mathrm{m}$, respectively. The chemical analysis of the ore sued is presented in Table 1 [5].

Table 1: Average analysis of the copper ore used in the study

\begin{tabular}{|l|c|c|c|c|c|c|}
\hline $\begin{array}{l}\text { Particle } \\
\text { size } \boldsymbol{\mu m}\end{array}$ & $\begin{array}{c}\mathbf{C u} \\
\mathbf{\%}\end{array}$ & $\begin{array}{c}\mathbf{M n} \\
\mathbf{\%}\end{array}$ & $\begin{array}{c}\mathbf{C a} \\
\mathbf{\%}\end{array}$ & $\begin{array}{c}\mathbf{F e} \\
\mathbf{\%}\end{array}$ & $\begin{array}{c}\mathbf{A l} \\
\mathbf{\%}\end{array}$ & $\begin{array}{c}\text { Density } \\
\mathbf{g} \mathbf{c m}^{\mathbf{3}}\end{array}$ \\
\hline $\mathbf{4 6 2}$ & 4.06 & 0.601 & 0.235 & 0.136 & 0.364 & 2.55 \\
\hline $\mathbf{1 1 0 0}$ & 5.05 & 0.578 & 0.106 & 0.163 & 0.395 & 2.55 \\
\hline $\mathbf{1 8 5 0}$ & 8.36 & 0.47 & 0.082 & 0.203 & 0.503 & 2.57 \\
\hline
\end{tabular}

The table also shows the densities of the ore particles. Analytical grade hydrochloric acid of concentration of 37\% was used to prepare different concentrations of $0.05,0.1$, and $0.2 \mathrm{~N}$.

\subsection{Studied Variables}

The effects of stirring speed, solid-to-liquid ratio, leaching temperature, acid concentration, and particle size, on copper leaching kinetics were examined. The stirring speed varied between 700 and $1000 \mathrm{rpm}$, while three leaching temperatures $\left(25,35\right.$, and $\left.45^{\circ} \mathrm{C}\right)$ were performed to reveal the effect of the temperature on the leaching kinetics. The solid to liquid ratios used were $1.0,2.0$, and $4.0 \mathrm{~g} / \mathrm{L}$ and to investigate the effect of the particle size, 462,1100 , and $1850 \mu \mathrm{m}$ particles were used.

\subsection{Experimental Procedure}

For a given experiment, one liter of the hydrochloric acid solution is prepared with the desired normality then poured into the reaction vessel and placed in a water bath which was heated and water circulated until the desired reaction temperature was attained. When the reaction solution equilibrated at the desired temperature the solution in the reaction vessel was agitated, at the desired stirring speed. An accurately weighed sample of the ore of the required particle size was transferred into the reactor, at the same time of pressing the start button of a stopwatch. Samples from the reaction vessel were removed at different predetermined times using a sampling device. Each sample was poured into a marked flask, stoppered, and kept for elemental analysis (copper and other elements) by the atomic absorption technique.

\section{RESULTS AND DISCUSSIONS}

The influence of stirring speed, solid-to-liquid ratio, leaching temperature, acid concentration, particle size, and acid consumption on the rate of leaching of copper from its ores were presented and discussed in detail in the first article of this series [6]. The kinetics and fitting of the leaching process will be presented and discussed below.

To examine the validity of the available heterogeneous reaction models for copper leaching from solid ores, two models were tested for analysis; the most widely used one for the analysis of solid-liquid reactions for unchanging size (SCM), and the mixed kinetics model.

\subsection{Shrinking Core Model Analysis}

During the experimental part and to minimize the resistance of the diffusion through the boundary layer, all the experiments were carried out at a stirring speed of $100 \mathrm{rpm}$, and hence, the boundary layer controlling step will not be taken into consideration in the analysis of the shrinking core model. As a result of this simplification, only the pore diffusion (Eq. 3), surface reaction (Eq. 5), and mixed kinetics conversion functions (Eq. 7) were considered for the purpose of modeling. Table 2 shows a sample of calculation estimated for the reaction of $462 \mu \mathrm{m}$ ore particles at a reaction temperature of $25{ }^{\circ} \mathrm{C}$ and $0.05 \mathrm{~N}$ of hydrochloric acid at a stirring speed of $1000 \mathrm{rpm}$ and a solid to liquid ratio of 1.0 $\mathrm{g} / \mathrm{L}$.

Table 2: Estimation of the surface reaction and pore diffusion terms for the reaction of $462 \mu \mathrm{m}$ at $25^{\circ} \mathrm{C}$ and $0.05 \mathrm{~N} \mathrm{HCl}$.

\begin{tabular}{|c|c|c|c|}
\hline $\begin{array}{l}\text { Time } \\
(\mathrm{min})\end{array}$ & $\begin{array}{c}\mathbf{X}_{\mathrm{Cu}} \\
\%\end{array}$ & $\underset{1-(1-X)^{1 / 3}}{\mathbf{A}}$ & $\begin{array}{c}\mathrm{B} \\
1-(2 / 3) X-(1-X)^{2 / 3}\end{array}$ \\
\hline 0.25 & 2.04 & 0.0068 & $4.666 \times 10^{-5}$ \\
\hline 0.5 & 3.51 & 0.0118 & $1.391 \times 10^{-4}$ \\
\hline 1 & 5.34 & 0.0181 & $3.246 \times 10^{-4}$ \\
\hline 1.5 & 7.15 & 0.0244 & $5.869 \times 10^{-4}$ \\
\hline 2 & 8.6 & 0.0295 & $8.549 \times 10^{-4}$ \\
\hline 3 & 11.11 & 0.0385 & $1.444 \times 10^{-3}$ \\
\hline 5 & 15.75 & 0.0555 & $2.969 \times 10^{-3}$ \\
\hline 10 & 25.34 & 0.0928 & $8.082 \times 10^{-3}$ \\
\hline 15 & 31.7 & 0.1193 & $1.311 \times 10^{-2}$ \\
\hline 20 & 36.61 & 0.1410 & $1.801 \times 10^{-2}$ \\
\hline 30 & 43.59 & 0.1737 & $2.669 \times 10^{-2}$ \\
\hline 40 & 48.1 & 0.1964 & $3.351 \times 10^{-2}$ \\
\hline 60 & 57.99 & 0.2511 & $5.248 \times 10^{-2}$ \\
\hline 90 & 59.36 & 0.2593 & $5.561 \times 10^{-2}$ \\
\hline 120 & 61.75 & 0.2741 & $6.140 \times 10^{-2}$ \\
\hline
\end{tabular}

Figure 9 shows the plots of the surface reaction rate function, $1-(1-X)^{1 / 3}$, versus leaching time $(t)$. If the surface 
reaction controls the reaction, a straight line with a slope of $\frac{v_{B} K_{S} C_{A_{L}}}{\rho_{B} R_{0}}$ should be obtained. The curves show straight lines only for the first few minutes, and after that, the curves are concave downwards and this indicates that surface reaction does not control the leaching step and hence does not fit the experimental data.

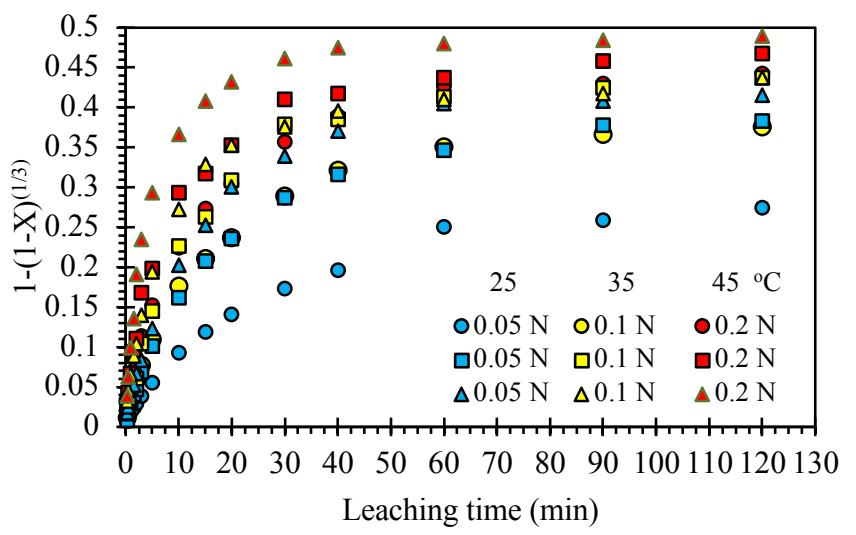

Figure 9: Surface reaction rate as a function of temperature and $\mathrm{HCl}$ concentration for particles of $462 \mu \mathrm{m}$ agitated at $1000 \mathrm{rpm}$

Plots of the pore diffusion conversion function $1-\frac{2}{3} X-$ $(1-X)^{2 / 3}$ against time are presented in Figure 10. If the pore diffusion controls the reaction, a straight line with a slope of $\frac{2 v_{B} D_{e} C_{A L}}{\rho_{B} R_{O}^{2}}$ should be obtained. Again, the curves show straight lines only for the first few minutes before they deviate from linearity. As a conclusion, pore diffusion as a controlling step does not fit the experimental data.

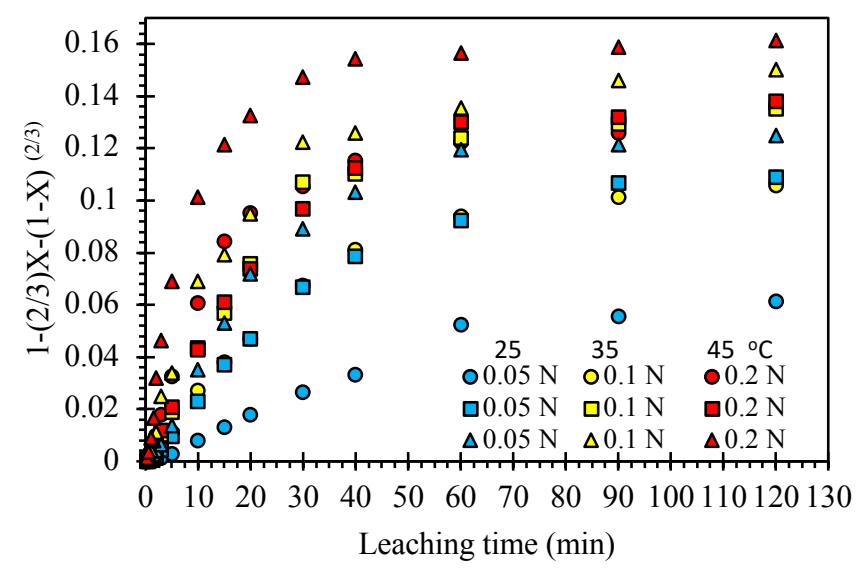

Figure 10: Product layer diffusion rate as a function of temperature and $\mathrm{HCl}$ concentration for particles of $462 \mu \mathrm{m}$.

By changing the particle size of the reacted ore, similar observations were detected for both the surface reaction rate and the pore diffusion conversion as demonstrated in Figures 11 and 12. As a result of this analysis, it is concluded that the shrinking core model is not a suitable model to describe the copper leaching process from this ore.

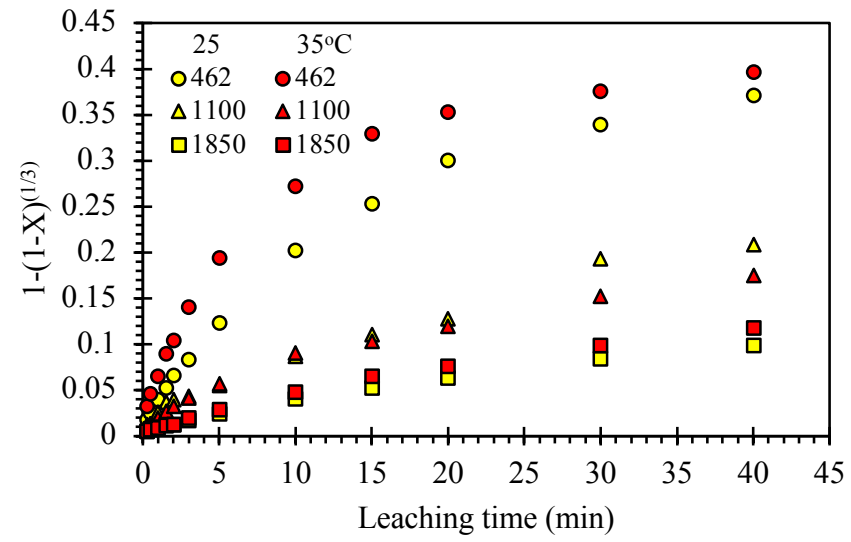

Figure 11: Surface reaction rate as a function of temperature and particle size leached in $0.2 \mathrm{~N} \mathrm{HCl}$ and agitated at 1000 rpm

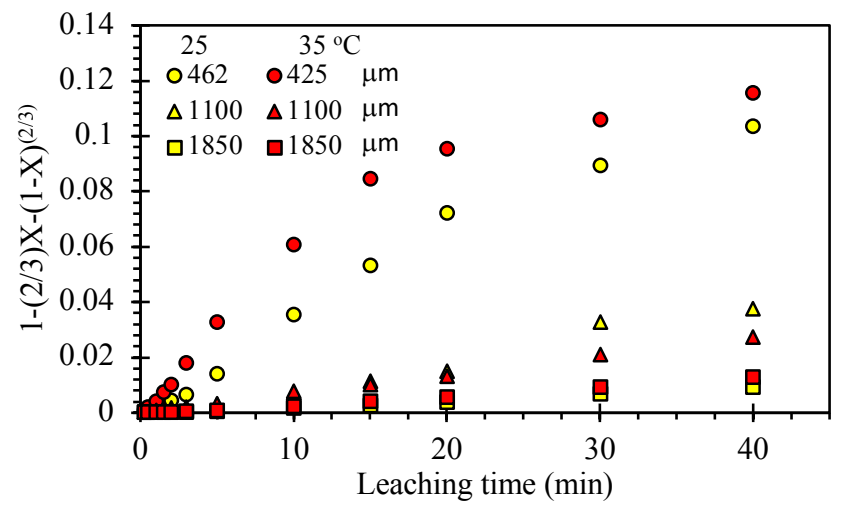

Figure 12: Product layer diffusion as a function of temperature and particle size leached in $0.2 \mathrm{~N} \mathrm{HCl}$ and agitated at $1000 \mathrm{rpm}$

\subsection{Mixed Kinetics Model Analysis}

Recall Eq. (7) which represents the final form of the mixed kinetics model

$$
1-\frac{2}{3} X-(1-X)^{\frac{2}{3}}+\beta\left[1-(1-X)^{\frac{1}{3}}\right]=\gamma C t
$$

the model has two unknown parameters $\beta$ and $\gamma$, these parameters can be found using the least-squares method. The above equation can be written as:

$A+\beta B=\gamma^{\prime} t$ where $\gamma^{\prime}=\gamma C$

where: $A=1-\frac{2}{3} X-(1-X)^{\frac{2}{3}}$ and $B=1-(1-X)^{\frac{1}{3}}$

$A$ and $B$ are functions of $X$ and, thus being the outcome of experimental measurements are subject to experimental errors. $A$ and $B$ are random variables (actually functions of $X$ ), and $t$ is an independent variable (to be fixed by the experimenter) and is measurable with negligible error. Thus, Eq. (8) can be represented as:

$A_{i}+\beta B_{i}+\varepsilon_{i}=\gamma^{\prime} t_{i}$

where $\varepsilon_{i}$ is the random error corresponding to each pair of observations $A_{i}$ and $B_{i}$. For every value of $t$ there is a probability of error $\varepsilon$ corresponding to effects of the errors in $A$ and $B$. Thus, values of parameters $\beta$ and $\gamma^{\prime}$ should be calculated based on the least-squares technique. Applying the least-squares procedure gives: 
$E=\sum_{i}^{n} \varepsilon_{i}^{2}=\sum_{i}^{n}\left(\gamma^{\prime} t_{i}-A_{i}-\beta B_{i}\right)^{2}$

where $n$ is the number of measurements. $E$ can be minimized by calculating its partial derivatives with respect to the unknown parameters $\beta$ and $\gamma^{\prime}$, equating each to zero, and solving simultaneously:

$\frac{\partial E}{\partial \beta}=0=\sum 2\left(\gamma^{\prime} t_{i}-A_{i}-\beta B_{i}\right) B_{i}$

$\frac{\partial E}{\partial \gamma^{\prime}}=0=\sum 2\left(\gamma^{\prime} t_{i}-A_{i}-\beta B_{i}\right) t_{i}$

These two equations can be arranged into:

$\gamma^{\prime} \sum B_{i} t_{i}-\sum A_{i} B_{i}-\beta \sum B_{i}^{2}=0$

$\gamma^{\prime} \sum t_{i}^{2}-\sum A_{i} t_{i}-\beta \sum B_{i} t_{i}=0$

Rearrange these equations:

$\gamma^{\prime} \sum B_{i} t_{i}-\beta \sum B_{i}^{2}=\sum A_{i} B_{i}$

$\gamma^{\prime} \sum t_{i}^{2}-\beta \sum B_{i} t_{i}=\sum A_{i} t_{i}$
The terms: $\sum B_{i} t_{i}, \sum B_{i}^{2}, \sum A_{i} B_{i}, \sum t_{i}^{2}, \sum A_{i} t_{i}$ are all constants and hence the above equations are a system of linear equations with $\beta$ and $\gamma^{\prime}$ are the unknown parameters. To obtain the values of $\beta$ and $\gamma^{\prime}$, one can solve the following system of linear equations by any numerical technique:

$\left[\begin{array}{cc}\sum B_{i} t_{i} & -\sum B_{i}^{2} \\ \sum t_{i}^{2} & -\sum B_{i} t_{i}\end{array}\right]\left[\begin{array}{l}\gamma^{\prime} \\ \beta\end{array}\right]=\left[\begin{array}{l}\sum A_{i} B \\ \sum A_{i} t_{i}\end{array}\right]$

Once the parameters of $\beta$ and $\gamma$ are calculated, then the values of $D$ and $K_{S}$ can be estimated using $K_{S}=\frac{2 D}{R_{0} \beta}$ and $D=$ $\frac{\gamma R_{0}^{2} v_{B}}{2}$. The calculated mixed kinetics parameters $\beta, \gamma, K_{S}$, and $D$ for all experiments performed in the current study are summarized in Table 3. The values of $D$ and $K_{S}$ were estimated using a value of $v_{B}=0.5$ for the number of moles of metal reacted per mole of $\mathrm{HCl}$.

Figures 13 shows the coupled effect of surface reaction and pore diffusion as a function of $\mathrm{HCl}$ for copper ore particles of

Table 3: Mixed kinetics parameters for copper dissolution using different hydrochloric acid concentration, leaching temperatures, and particle size at a stirring speed is $1000 \mathrm{rpm}$ and a solid to liquid ratio of $1.0 \mathrm{~g} / \mathrm{L}$.

\begin{tabular}{|c|l|c|c|c|c|c|}
\hline $\begin{array}{c}\text { size } \\
\boldsymbol{\mu m}\end{array}$ & $\begin{array}{c}\mathbf{H C l} \\
\mathbf{N}\end{array}$ & $\begin{array}{c}\text { Temperature } \\
\mathbf{}^{\mathbf{C}}\end{array}$ & $\begin{array}{c}\boldsymbol{\beta} \\
(\mathbf{d i m e n s i o n l e s s})\end{array}$ & $\begin{array}{c}\boldsymbol{\gamma} \\
(\mathbf{L} / \mathbf{m o l} . \mathbf{s e c})\end{array}$ & $\begin{array}{c}\boldsymbol{D} \\
(\mathbf{c m} / \mathbf{s e c})\end{array}$ & $\begin{array}{c}\boldsymbol{K} \boldsymbol{S} \\
(\mathbf{c m} / \mathbf{s e c})\end{array}$ \\
\hline 462 & 0.05 & 25 & $9.5921 \times 10^{-3}$ & $3.0474 \times 10^{-4}$ & $4.0653 \times 10^{-8}$ & $3.6694 \times 10^{-4}$ \\
\hline & 0.1 & 25 & $6.6179 \times 10^{-3}$ & $3.9305 \times 10^{-4}$ & $5.2434 \times 10^{-8}$ & $6.8598 \times 10^{-4}$ \\
\hline & 0.2 & 25 & $7.1777 \times 10^{-2}$ & $3.9431 \times 10^{-4}$ & $5.2602 \times 10^{-8}$ & $6.3450 \times 10^{-5}$ \\
\hline & 0.05 & 35 & $1.7318 \times 10^{-3}$ & $8.2390 \times 10^{-4}$ & $1.0991 \times 10^{-7}$ & $5.4949 \times 10^{-3}$ \\
\hline & 0.1 & 35 & $3.2106 \times 10^{-3}$ & $6.5078 \times 10^{-4}$ & $8.6816 \times 10^{-8}$ & $2.3412 \times 10^{-3}$ \\
\hline & 0.2 & 35 & $1.7181 \times 10^{-2}$ & $5.5367 \times 10^{-4}$ & $7.3861 \times 10^{-8}$ & $3.7221 \times 10^{-4}$ \\
\hline & 0.05 & 45 & $1.4613 \times 10^{-2}$ & $1.4776 \times 10^{-3}$ & $1.9712 \times 10^{-7}$ & $1.1679 \times 10^{-3}$ \\
\hline & 0.1 & 45 & $5.4071 \times 10^{-3}$ & $1.1806 \times 10^{-3}$ & $1.5749 \times 10^{-7}$ & $2.5219 \times 10^{-3}$ \\
\hline & 0.2 & 45 & $4.7816 \times 10^{-3}$ & $1.2016 \times 10^{-3}$ & $1.6030 \times 10^{-7}$ & $2.9024 \times 10^{-3}$ \\
\hline 1100 & 0.1 & 25 & $1.6905 \times 10^{-3}$ & $4.7404 \times 10^{-5}$ & $3.5849 \times 10^{-8}$ & $7.7114 \times 10^{-4}$ \\
\hline & 0.2 & 25 & $6.7483 \times 10^{-3}$ & $8.2076 \times 10^{-5}$ & $6.2070 \times 10^{-8}$ & $3.3447 \times 10^{-4}$ \\
\hline & 0.2 & 35 & $4.7202 \times 10^{-3}$ & $5.9183 \times 10^{-5}$ & $4.4757 \times 10^{-8}$ & $3.4480 \times 10^{-4}$ \\
\hline & 0.2 & 45 & $1.5315 \times 10^{-2}$ & $1.0195 \times 10^{-4}$ & $7.710-0 \times 10^{-8}$ & $1.8306 \times 10^{-4}$ \\
\hline 1850 & 0.1 & 35 & $1.8643 \times 10^{-2}$ & $2.9807 \times 10^{-5}$ & $6.3759 \times 10^{-8}$ & $7.3946 \times 10^{-5}$ \\
\hline & 0.2 & 25 & $2.5947 \times 10^{-3}$ & $1.9345 \times 10^{-5}$ & $4.1380 \times 10^{-8}$ & $3.4482 \times 10^{-4}$ \\
\hline & 0.2 & 35 & $6.8464 \times 10^{-3}$ & $2.7652 \times 10^{-5}$ & $5.9149 \times 10^{-8}$ & $1.8680 \times 10^{-4}$ \\
\hline & 0.2 & 45 & $4.2876 \times 10^{-2}$ & $1.1034 \times 10^{-4}$ & $2.3602 \times 10^{-7}$ & $1.1902 \times 10^{-4}$ \\
\hline
\end{tabular}

$462 \mu \mathrm{m}$ reacted at $25^{\circ} \mathrm{C}$ and agitated at $1000 \mathrm{rpm}$. The coupled effect is formulated in terms of $X$ and $\beta$ of Eq. (7) as: $1-\frac{2}{3} X-(1-X)^{\frac{2}{3}}+\beta\left[1-(1-X)^{\frac{1}{3}}\right]$, and in all these curves a linear plot passes through the zero coordinates is obtained. Beyond the leaching time indicated for each curve, the lines deviate from linearity at a copper dissolution values of 58,64 , and $66 \%$ for $\mathrm{HCl}$ concentration of $0.05,0.1$, and 2.0 respectively.

For other leaching temperatures and acid concentrations of the same ore particle size of $462 \mu \mathrm{m}$, similar trends can be observed as presented in Figure 14. By increasing the ore particle size, mixed kinetics curves also follow the same trend as presented in Figure 15. As a result of the above analysis, it can be concluded that Eq. (7) is a generalized equation which describes coupled diffusion and surface reaction kinetics, whereas:

$k_{p} t=1-(1-X)^{2 / 3}-\frac{2}{3} X$

And $k_{S} t=1-(1-X)^{1 / 3}$

are asymptotic solutions in the sense that as $\beta \rightarrow 0$ Eq. (7) tends to Eq. (18) and as $\beta \rightarrow \infty$ (large value) Eq. (7) tends to Eq. (19). 


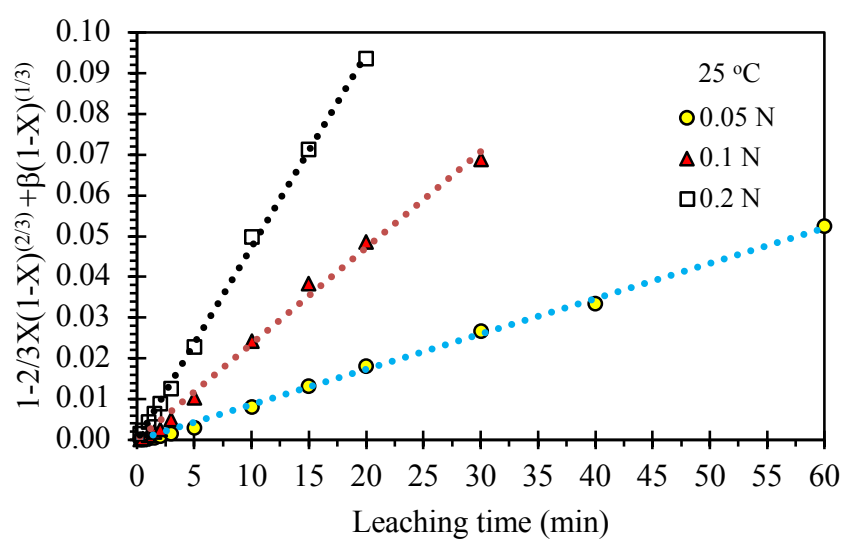

Figure 13: Mixed kinetics model as a function of $\mathrm{HCl}$ concentration for reacted particles of $462 \mu \mathrm{m}$ at $25^{\circ} \mathrm{C}$ and a stirring speed of 1000 rpm.

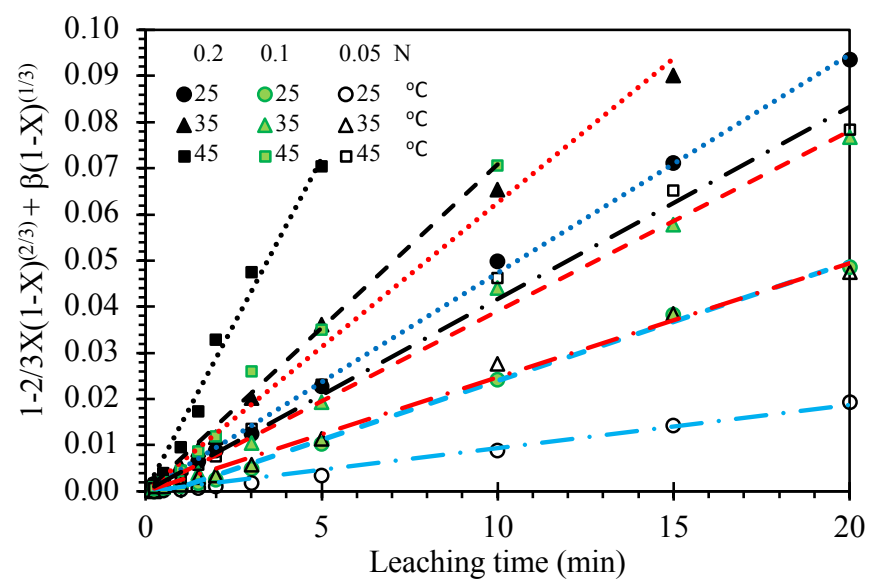

Figure 14: Mixed kinetics model as a function of leaching temperature and $\mathrm{HCl}$ concentration for ore particles of 462 $\mu \mathrm{m}$ agitated at a stirring speed of $1000 \mathrm{rpm}$

It can be said that the mixed kinetics model can fit the copper leaching experimental data up to at least $65 \%$ conversion, but after this conversion, the values obtained from the model deviate from the experimental values, as shown in Figure 16 where the operating conditions for $R_{1}, R_{2}$, and $R_{3}$ are summarized in Table 4.

The problem of leveling off has been observed in the work of Bryden [15] and Al-Sayyed [23]. In a Kinetics study of chalcopyrite leaching by sodium nitrate in sulfuric acid carried out by Sokic et al. [24], the kinetic data obtained for copper leaching showed a good fit to the mixed control model.

Saxena and Mandre [25] studied the kinetics of copper dissolution using ferric chloride and observed that chemical reaction at the mineral surface is rate controlling in the initial stages and, during later stages, diffusion through the product layer is rate-controlling, the overall reaction is described by the mixed control model.

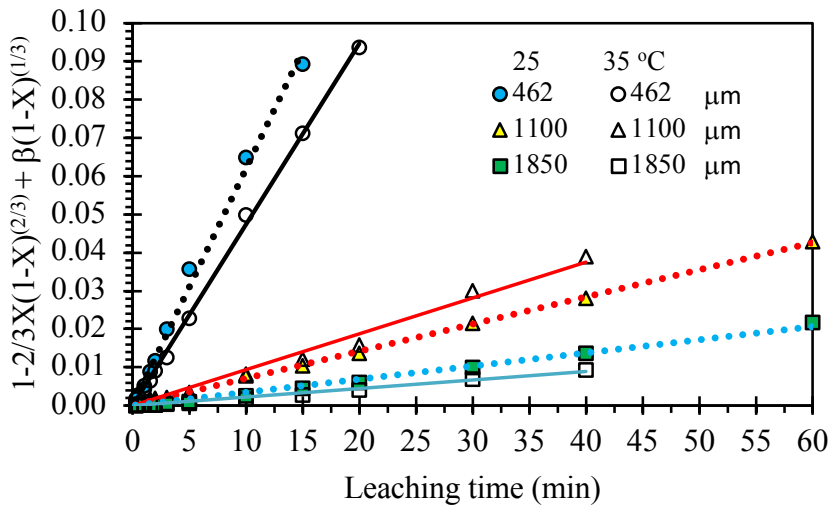

Figure 15: Mixed kinetics model as a function of leaching temperature and ore particle size reacted in a medium of $0.2 \mathrm{~N}$ $\mathrm{HCl}$ at a stirring speed of $1000 \mathrm{rpm}$.

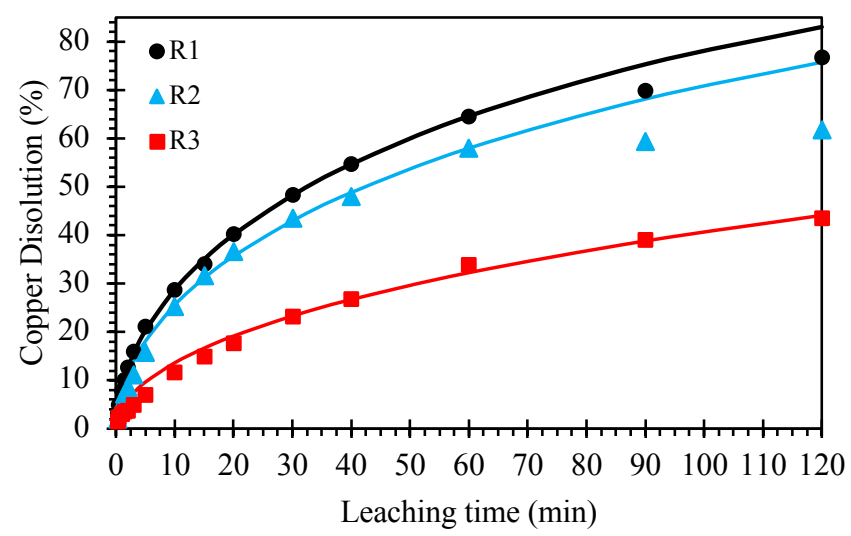

Figure 16: Comparison between experimental (symbols) and mixed kinetics model (line) copper dissolution for different runs.

Table 4: Operating conditions for runs $\mathrm{R}_{1}, \mathrm{R}_{2}$, and $\mathrm{R}_{3}$

\begin{tabular}{|c|c|c|c|c|c|}
\hline $\begin{array}{c}\text { Run } \\
\text { No. }\end{array}$ & $\begin{array}{c}\mathbf{T} \\
\left({ }^{\mathbf{}} \mathbf{C}\right)\end{array}$ & $\begin{array}{c}\text { HCl } \\
(\mathbf{N})\end{array}$ & $\begin{array}{c}\text { Speed } \\
(\mathbf{r p m})\end{array}$ & $\begin{array}{c}\text { Particle } \\
(\boldsymbol{\mu} \mathbf{m})\end{array}$ & $\begin{array}{c}\text { S/L } \\
(\mathbf{g} / \mathbf{L})\end{array}$ \\
\hline R1 & 45 & 0.2 & 1000 & 1100 & 1.0 \\
\hline R2 & 25 & 0.05 & 1000 & 462 & 1.0 \\
\hline R3 & 25 & 0.2 & 1000 & 1850 & 1.0 \\
\hline
\end{tabular}

\subsection{Mixed Kinetics Parameters}

The values of $D$ obtained in this work and tabulated in Table 3 are in the range of $3.58 \times 10^{-8}$ to $2.36 \times 10^{-7} \mathrm{~cm}^{2} / \mathrm{s}$. Madsen et al. [26] reported values of $D$ in the range of $2 \times 10^{-7}$ to $6.3 \times 10^{-}$ ${ }^{7} \mathrm{~cm}^{2} / \mathrm{s}$. Bryden [15] obtained values of $D$ in the range of $3.5 \times 10^{-10}$ to $6.5 \times 10^{-6} \mathrm{~cm}^{2} / \mathrm{s}$, and Al-Sayyed [23] found values of $D 1.9 \times 10^{-8}$ to $1.3 \times 10^{-6} \mathrm{~cm}^{2} / \mathrm{s}$. Thus, the present values of $D$ are close to the values of the previous studies. Bryden [15] reported values in the range of $7.6 \times 10^{-7}$ to $2.5 \times 10^{-2} \mathrm{~cm} / \mathrm{s}$ for $K_{S}$, and Al-Sayyed [23] found values of $K_{S}$ in the range of $5 \times 10^{-5}$ to $1.8 \times 10^{-3} \mathrm{~cm} / \mathrm{s}$. In this study, the values of $K_{S}$ obtained are in the range of $6.34 \times 10^{-5}$ to $5.49 \times 10^{-3} \mathrm{~cm} / \mathrm{s}$, thus the values obtained in the present work are close to those reported in the literature.

\subsection{Effect of Temperature on $K_{S}$ and $D$}

As previously shown [6], copper dissolution increases as the leaching temperature increases. Table 3 shows the effect of 
leaching temperature on the pore diffusion coefficients $(D)$ and surface reaction constants $\left(K_{S}\right)$, increasing leaching temperature results in an increase in $D$ and $K_{S}$ and this can be explained by the Eyring equation, $D=D_{o} e^{\left(\frac{-E_{p}}{R T}\right)}$, and Arrhenius equation, $K_{S}=A_{o} e^{\left(\frac{-E_{S}}{R T}\right)}$. Rewrite these equations: Eyring equation becomes $-\ln (D)=-\ln \left(D_{o}\right)+\frac{E_{p}}{R} \frac{1}{T}$ and Arrhenius equation becomes $-\ln \left(K_{S}\right)=-\ln \left(A_{o}\right)+\frac{E_{S}}{R} \frac{1}{T}$, and plotting $-\ln (D)$ vs. $1 / T$ will give a straight line with a slope of $\frac{E_{p}}{R}$ and plotting $-\ln \left(K_{S}\right)$ vs. $1 / T$ will give a straight line with a slope of $\frac{E_{S}}{R}$.

Figure 17 represents these two relations graphically, multiplying the obtained slope with the gas constant $\left(1.987 \times 10^{-3} \mathrm{kcal} \cdot \mathrm{K}^{-1} \cdot \mathrm{mol}^{-1}\right)$ gives the activation energies. An average value of pore diffusion activation energy $\left(E_{p}=10.34\right.$ $\mathrm{kcal} / \mathrm{mole})$ and the chemical reaction activation energy $\left(E_{s}=\right.$ $12.37 \mathrm{kcal} / \mathrm{mole}$ ) were obtained for ore particles of $462 \mu \mathrm{m}$ reacted in $0.1 \mathrm{~N} \mathrm{HCl}$ at a stirring speed of $1000 \mathrm{rpm}$.

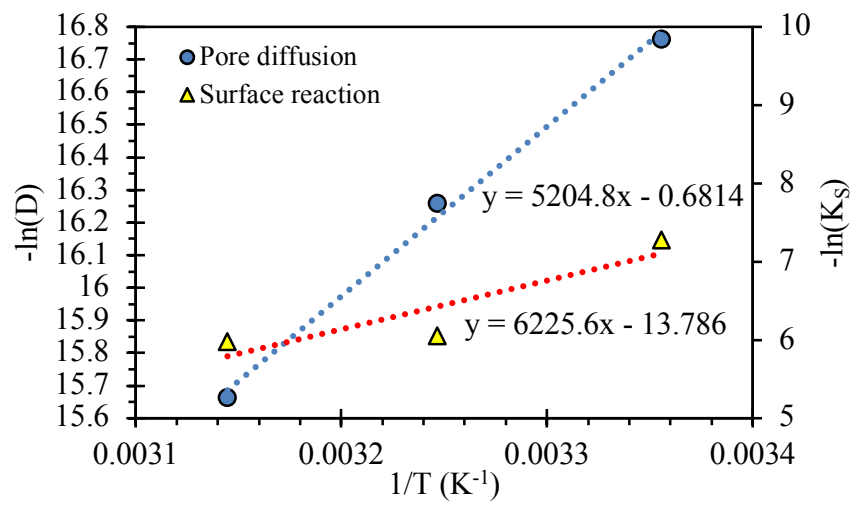

Figure 17: Determination of pore diffusion and chemical reaction activation energies for copper ore particles of $462 \mu \mathrm{m}$ reacted in $0.1 \mathrm{~N} \mathrm{HCl}$ at a stirring speed of $1000 \mathrm{rpm}$.

The values obtained in the current work are closer to those reported by Bryden [15] who reported values for $E_{p}$ in the range of 7.55 to $10.11 \mathrm{kcal} / \mathrm{mole}$ and $E_{s}$ range of 3.88 to 13.61 $\mathrm{kcal} / \mathrm{mol}$. The average activation energy of copper leaching from low-grade cuprite ores was determined to be $45.28 \mathrm{~kJ}$ mol-1 by Bai et al. [16]. Azizi et al. [17] estimated a value of $26.699 \mathrm{~kJ} /$ mole for the dissolution kinetics of copper oxide ore in sulfuric acid. In their study, Tanaydin and Demirkiran [18] reported an activation energy value of $34.69 \mathrm{~kJ} / \mathrm{mole}$ for the leaching kinetics of malachite in perchloric acid solutions. Ghosh et al. [21] claimed a value of $37.6 \pm 1.9 \mathrm{~kJ} / \mathrm{mol}$ for ammonia leaching kinetics of complex copper ore.

\section{CONCLUSIONS}

In this study, the leaching kinetics of a Jordanian copper ore was studied using hydrochloric acid solutions as the lixiviant. The effects of several parameters on copper leaching from the ore were examined, and their effects on the kinetics were made. Reaction kinetics of hydrochloric acid leaching of the ore was described by the coupled diffusion-chemical reaction equation (mixed kinetics model) up to $65 \%$ copper dissolution, and the process was characterized by two apparent activation energies for pore diffusion and surface reaction. The values obtained for $K_{S}$ and $D$ for hydrochloric acid leaching are $K_{S}=6.34 \times 10^{-4}-5.49 \times 10^{-3} \mathrm{~cm} / \mathrm{sec}$ and $D=$ $3.58 \times 10^{-8}-2.36 \times 10^{-7} \mathrm{~cm}^{2} / \mathrm{s}$, while the apparent activation energies for pore diffusion and surface reaction were estimated to be 12.26 and $9.61 \mathrm{kcal} / \mathrm{mol}$, respectively.

\section{REFERENCES}

1. N. Habbache, N. Alane,S. Djerad. Leaching of copper oxide with different acid solutions, Chemical Engineering Journal, Vol. 152(2-3), pp.503-508, 2009. https://doi.org/10.1016/j.cej.2009.05.020

2. M.E. Arzutug, M.M. Kocakerim, M. Copur. Leaching of malachite ore in NH3-saturated water,Industrial \& Engineering Chemistry Research, Vol. 43(15), pp.41184123, 2004.

https://doi.org/10.1021/ie0342558

3. O. Hyvärinen, M. Hämäläinen. HydroCopper ${ }^{\text {TM }}$ - a new technology producing copper directly from concentrate, Hydrometallurgy, Vol. 77(1-2), pp.61-65, 2005.

https://doi.org/10.1016/j.hydromet.2004.09.011

4. A. Ekmekyapar, E. Aktas, A. Kunkul and N. Demirkiran. Investigation of leaching kinetics of copper from malachite ore in ammonium nitrate solution, Metallurgical and Materials Transactions B, Vol. 43, pp.764-772, 2012.

https://doi.org/10.1007/s1 1663-012-9670-2

5. Y. Mubarak. Hydrochloric Acid Leaching of Jordanian Copper Ores, M.Sc. Thesis, University of Jordan, Amman, Jordan, 1992.

6. Y. Mubarak. Leaching of Copper Ores: Effects of Operating Variables, International Journal of Emerging Trends in Eng. Research, Vol. 8(8), pp.4226-4235, 2020. https://doi.org/10.30534/ijeter/2020/31882020

7. O. Levenspiel. Chemical Reaction Engineering, $2^{\text {nd }} E d$., John Wiley and Sons Inc., New York, USA, 1972.

8. L.K. Doraiswamy, M.M. Sharma. Heterogeneous Reactions - Analysis, Examples, and Reactor Design, Vol. 1, John-Wiley and Sons, New York, USA, 1983.

9. S. Yagi, D. Kunii. Studies on Effective Thermal Conductivities in Packed Beds, A.I.Ch.E. Journal, Chemical Engineering (Japan), Vol. 3(3), pp.373-381, 1957. https://doi.org/10.1002/aic.690030317

10. L.K. Doraiswamy, B.D. Kulkarni. Gas-solid noncatalytic reactions, Chapter 5, in Chemical Reaction and Reaction Eng., Dekker, New York, USA, 1987.

11. O. Levenspiel. The Chemical Reaction Omnibook, O.S.U. Book Stores, Corvallis, USA, 1979.

12. J.Y. Park, O. Levenspiel. The Crackling Core Model for the Reaction of Solid Particles, Chemical Eng. 
Science, Vol. 30, pp.1207-1214, 1975 https://doi.org/10.1016/0009-2509(75)85041-X

13. P.C. Hsu, L Ep. Murr. Simple Kinetic Model for Sulfuric Acid Leaching of Copper from Chrysocolla, Metallurgical Transactions B, Vol 6B, pp.435-440, 1975. https://doi.org/10.1007/BF02913829

14. M.E. Wadsworth. Kinetics of Heterogeneous System, Annu. Rev. Phys. Chem., Vol. 23, pp.355-384, 1972.

15. K.O. Bryden. Ammonium-Sulfate Leaching of Malachite. A Kinetic Study, Ph.D. Thesis, University of Utah, USA, 1980.

16. S. Bai, X. Fu, C. Li,S. Wen. Process improvement and kinetic study on copper leaching from low-grade cuprite ores, Physicochemical Problems of Mineral Processing, 54(2), pp.300-310, 2018. http://dx.doi.org/10.5277/ppmp1818

17. B. Bayati, A. Azizi, M. Karamoozian. A comprehensive study of the leaching behavior and dissolution kinetics of copper oxide ore in sulfuric acid lixiviant. Scientia Iranica, 25(3), pp.1412-1422, 2018. DOI: $10.24200 /$ SCI.2018.5226.1154

18. M.K. Tanaydin, N. Demirkiran. Investigation of selective leaching and kinetics of copper from malachite ore in aqueous perchloric acid solutions. Separation Science and Technology, Vol. 54(5), pp.815827, 2019. https://doi.org/10.1080/01496395.2018.1512619

19. M. K. Tanaydin, N. Demirkıran, A. Ekmekyapar. Kinetic Model of Copper Leaching from a Malachite Ore by Acetic Acid Solution, Journal of Chemical Technology and Metallurgy, Vol. 55(4), pp.852-859, 2020.

20. F.W. Ntengwe. The Leaching of Dolomitic-Copper Ore Using Sulphuric Acid under Controlled Conditions, The Open Mineral Processing Journal, Vol. 3, pp.60-67, 2010.

DOI: $10.2174 / 1874841401003010060$

21. A.A. Baba, M.K. Ghosh, S.R. Pradhan, D.S. Rao, A. Baral, F.A. Adekola. Characterization and kinetic study on ammonia leaching of complex copper ore, Transaction of Nonferrous Metals Society China, Vol. 24, pp.1587-1595, 2014. https://doi.org/10.1016/S1003-6326(14)63229-5

22. I.G. Reilly,D.S. Scott. The leaching of a chalcopyrite concentrate in ammonia. Canadian Journal of Chemical Engineering, Vol. 55, pp.527-533, 1977. https://doi.org/10.1002/cjce.5450550508

23. M.F. Al-Sayyed. Leaching of Copper from Wadi Araba Jordanian Ore, M.Sc. Thesis, University of Jordan, Amman, Jordan, 1988.

24. M.D. Sokić, B. Marković and D. Živković. Kinetics of chalcopyrite leaching by sodium nitrate in sulphuric acid, Hydrometallurgy, Vol. 95, pp.273-279, 2009. https://doi.org/10.1016/j.hydromet.2008.06.012

25. N.N. Saxena and N.R. Mandre. Mixed control kinetics of copper dissolution for copper ore using ferric chloride, Hydrometallurgy, Vol. 28, pp.111-117, 1992. https://doi.org/10.1016/0304-386X(92)90068-B

26. B.W. Madsen and M.E. Wadsworth. The Application of a Mixed Kinetics Leaching Model to an Ore Containing an Assemblage of Different Copper
Minerals, Proc. Am. Nuclear Soc., Topical Meeting on Energy and Mineral Resource Recovery, Colorado School of Mines, Golden, Colorado pp.619-630, 1977. 